RAD HAZU. MATEMATIČKE ZNANOSTI

Vol. $23=538(2019): 1-11$

DOI: https : //doi.org/10.21857/yq32oh4qd9

\title{
REARRANGING ABSOLUTELY CONVERGENT WELL-ORDERED SERIES IN BANACH SPACES
}

\author{
VEDRan ČaČIĆ, Marko DoKo AND Marko Horvat
}

\begin{abstract}
We identify a general reordering principle for well-ordered series in Banach spaces. We prove that for every absolutely convergent wellordered series indexed by a countable ordinal, if the series is rearranged according to any countable ordinal, then the absolute convergence and the sum of the series remain unchanged.
\end{abstract}

\section{INTRODUCTION}

Well-known results in mathematical anaysis tell us that whenever a series of real numbers is absolutely convergent, then it is possible to:

- reorder the terms by subjecting their indices to some permutation of $\omega$,

- split the series into an odd-indexed and an even-indexed part, sum each of the two parts separately, and add the results,

- arrange the terms into an $\omega \times \omega$ matrix, then sum each column, and then all the results, etc.

A natural question to ask is whether all these results are special cases of some general principle. Before we give a positive answer to this question, we need to discuss our choice of setting as well as the generalization of the notion of sequence reordering. For the former, we choose Banach spaces as convenient ambient spaces that allow us to compute sums, take their unique limits, measure them with the defined norm, and infer convergence from absolute convergence when needed. For the latter, sequences are typically reordered by composition with some bijection from $\omega$ to $\omega$. This means that each term of the reordered sequence is preceded by finitely many other terms. In this work, we consider bijections between $\omega$ and some ordinal number $\alpha$, where

2010 Mathematics Subject Classification. 03E10, 40A05.

Key words and phrases. Well-ordered series, summability, absolute convergence, reordering, Banach spaces, ordinals.

This work is supported by the Croatian Science Foundation, project no. 7459 (CompStruct). 
"sequence" terms might have infinite indices. For example, the three previously mentioned reorderings are cases where $\alpha$ is respectively $\omega, \omega \cdot 2$, and $\omega^{2}$.

The corresponding extension of the notion of series is known as wellordered series. We show that for every absolutely convergent well-ordered series indexed by a countable ordinal, if the series is rearranged according to some other countable ordinal, then the absolute convergence and the sum of the series remain unchanged.

In order to make our main contributions outlined in the previous paragraph fully precise, we provide a discussion of the related work as well as the necessary background in the following sections.

\section{RELATED WORK}

A plethora of results were being published throughout the 20th century that cover a vast range of topics at the intersection of set theory and analysis, including the absolute convergence of series in normed spaces $[2,3,6]$. Some publications include the study of well-ordered series in various contexts $[2$, $7,8]$, which even extends to recent years [5]. However, to the best of our knowledge, the effects of the reorderings outlined in the previous section on the absolute convergence of well-ordered series in Banach spaces have not yet been thoroughly explored.

In the book Foundations of modern analysis [2], Dieudonné defines absolutely convergent well-ordered series $\Sigma\left(a_{i}\right)_{i \in \alpha}$ in Banach spaces by considering the absolute convergence of the series $\Sigma\left(a_{f(i)}\right)_{i \in \omega}$ for any bijection $f: \omega \rightarrow \alpha$. The notion is well defined because it does not depend on the choice of bijection. This is also true for the notion of its sum, which can be lifted analogously. We choose more general definitions of well-ordered series, their convergence and sum, which do not depend on absolute convergence, and expand on the work of Dieudonné by investigating the relationship of differently indexed well-ordered series with the same terms.

\section{BACKGROUND}

We define ordinals in the von Neumann sense and use standard notation: $\omega$ is the set of all finite ordinals, $\omega_{1}$ is the set of all countable ordinals, and Lim is the class of all limit ordinals (those that are neither 0 nor successors).

A normed space $(X,\|\cdot\|)$ is a vector space $X$ equipped with a norm $\|\cdot\|$. We sometimes omit the norm and simply refer to $(X,\|\cdot\|)$ as $X$. If a normed space $X$ is complete, it is called a Banach space. For an ordinal $\beta \in \omega_{1} \backslash \omega$ and a normed space $X$, we call a function $a: \beta \rightarrow X$ a hypersequence ${ }^{1}$. We denote the value of a hypersequence $a$ at argument (index) $\alpha$ by $a_{\alpha}$. If we want

\footnotetext{
${ }^{1}$ Similar functions are sometimes called $\beta$-sequences [1] to make the index domain explicit.
} 
to emphasize the domain, we write $\left(a_{\alpha}\right)_{\alpha \in \beta}$ instead of $a$. Note that we only consider countably infinite domains because of the known fact that convergent series with uncountably many positive terms contain only countably many non-zero terms [4].

A common way to define the sum of a series involves limits of partial sum sequences. We define the notion of well-ordered series in an analogous fashion; we call the main building block a partial sum hypersequence.

Definition 3.1. Let $(X,\|\cdot\|)$ be a normed space. We say that the hypersequence $s: \gamma \rightarrow X$ is a partial sum hypersequence of a hypersequence $a: \beta \rightarrow X$, if the following conditions hold:

(1) $1 \leqslant \gamma \leqslant \beta+1$

(2) $s_{0}=0$

(3) $(\forall \alpha \in \gamma)\left(\alpha+1 \in \gamma \Rightarrow s_{\alpha+1}=s_{\alpha}+a_{\alpha}\right)$

(4) $(\forall \lambda \in \gamma)\left(\lambda \in \operatorname{Lim} \Rightarrow s_{\lambda}=\lim _{\alpha \rightarrow \lambda} s_{\alpha}\right)$

By the limit in condition (4), we mean

$$
\left(\forall \varepsilon \in \mathbb{R}^{+}\right)\left(\exists \alpha_{0} \in \lambda\right)(\forall \alpha \in \lambda)\left(\alpha \geqslant \alpha_{0} \Rightarrow\left\|s_{\lambda}-s_{\alpha}\right\|<\varepsilon\right) .
$$

Hypersequences are naturally partially ordered by restriction (as functions), which is the same as the subset relation when we consider functions as sets of ordered pairs.

Proposition 3.2. For every hypersequence a, there exists the largest partial sum hypersequence of a (that is, the one of which all other partial sum hypersequences of a are restrictions).

Proof. It is possible to show, by transfinite induction, that every two partial sum hypersequences of $a$ must agree on the intersection of their domains. Namely, the conditions from the definition prescribe the values of $s$ on 0 , successors and limit ordinals in its domain, using previous values (existing limits are unique because the codomain is a normed space, hence Hausdorff).

Moreover, because their domain is bounded from above, all partial sum hypersequences of $a$ form a set - a subset of $\mathcal{P}((\beta+1) \times X)$. That set is also nonempty, since it contains the trivial hypersequence $\langle 0\rangle$ with domain 1 . But then the union of that set is also a partial sum hypersequence of $a$, and it is obviously the largest one.

Since it is the largest, it is unique for every $a$, and we denote it by $\sigma a$. We can now give a precise definition of the notion of well-ordered series.

DeFinition 3.3. Let a be a hypersequence and $\sigma a$ its largest partial sum hypersequence. We define the well-ordered series $\Sigma a$ as the ordered pair $(a, \sigma a)$.

The concepts of convergence, divergence, and absolute convergence can be directly extended to well-ordered series. Before we list the definitions, we 
prove that for a given hypersequence $a$, there are two essentially different possibilities for the domain of $\sigma a$.

Proposition 3.4. For every hypersequence $a: \beta \rightarrow X$, the domain of $\sigma a$ is either $\beta+1$ or some limit ordinal not greater than $\beta$.

Proof. We denote the domain of $\sigma a$ by $\gamma$. The first condition of Definition 3.1 says that $\gamma$ is not zero, so it is either a successor or a limit ordinal.

- First assume $\gamma$ is a successor, different from $\beta+1$. The first condition of Definition 3.1 says that it cannot be larger than $\beta+1$, so it must be smaller: $\gamma=\delta+1 \in \beta+1$. But then $\delta \in \gamma$, which means that $(\sigma a)_{\delta}$ is defined, and also $\delta \in \beta$, which means $a_{\delta}$ is defined. Adding those two numbers, we can extend $\sigma a$ to its proper superset

$$
\sigma a \cup\left\{\left(\delta+1,(\sigma a)_{\delta}+a_{\delta}\right)\right\}
$$

which is also a partial sum hypersequence of $a$, contradicting $\sigma a$ being the largest one. So if $\gamma$ is a successor, it must be the successor of $\beta$.

- Now assume $\gamma$ is a limit ordinal. The first condition of Definition 3.1 implies that it is not greater than $\beta+1$, and it cannot be equal to $\beta+1$ because it is a limit ordinal. Therefore, it must be smaller than $\beta+1$, i.e., not greater than $\beta$.

DeFinition 3.5. Let $a: \beta \rightarrow X$ be a hypersequence. If the domain of $\sigma a$ is $\beta+1$, we say that the well-ordered series $\Sigma a$ converges. In that case, $(\sigma a)_{\beta} \in X$ is called the sum of $\Sigma a$, and is denoted by $\sum_{\alpha \in \beta} a_{\alpha}$. Otherwise, we say that the well-ordered series $\Sigma a$ diverges. We say that $\Sigma a$ absolutely converges if the well-ordered series $\Sigma\left(\left\|a_{\alpha}\right\|\right)_{\alpha \in \beta}$ converges.

It is straightforward to verify that standard series with real terms can be identified with well-ordered series over $\beta=\omega$, and the definitions of convergence and absolute convergence in that case coincide with our definitions. The domain $\gamma$ of each partial sum hypersequence $s$ of $a$ is in that case either $\omega$ (if the series diverges) or $\omega+1$ (if it converges). From this point on, we use the terms well-ordered series and series, and the notation $\Sigma\left(a_{i}\right)_{i \in \omega}$ and $\sum_{i=0}^{\infty} a_{i}$, interchangeably.

We now formulate and prove two simple lemmas that will be an often used tool in the following section. They are a direct result of the topological fact that limits commute with continuous functions, and they let us sum a series by first summing two disjoint parts, and then adding the two results together. We denote the characteristic function of $S \subseteq X$ by $\mathbf{1}_{S}$.

LEMma 3.6. Let $X$ be a normed space, $\left(a_{i}\right)_{i \in \omega}$ a sequence in $X$, and $S \subseteq \omega$. If the series $\Sigma\left(a_{i} \cdot \mathbf{1}_{S}(i)\right)_{i \in \omega}$ and $\Sigma\left(a_{i} \cdot \mathbf{1}_{\omega \backslash S}(i)\right)_{i \in \omega}$ converge, then the 
series $\Sigma\left(a_{i}\right)_{i \in \omega}$ also converges and

$$
\sum_{i=0}^{\infty} a_{i}=\sum_{i=0}^{\infty}\left(a_{i} \cdot \mathbf{1}_{S}(i)\right)+\sum_{i=0}^{\infty}\left(a_{i} \cdot \mathbf{1}_{\omega \backslash S}(i)\right) .
$$

Proof. We compute

$$
\begin{aligned}
\sum_{i=0}^{\infty} a_{i} & =\lim _{n} \sum_{i=0}^{n}\left(a_{i} \cdot\left(\mathbf{1}_{S}(i)+\mathbf{1}_{\omega \backslash S}(i)\right)\right) \\
& =\lim _{n}\left(\sum_{i=0}^{n}\left(a_{i} \cdot \mathbf{1}_{S}(i)\right)+\sum_{i=0}^{n}\left(a_{i} \cdot \mathbf{1}_{\omega \backslash S}(i)\right)\right) \\
& \stackrel{(*)}{=} \lim _{n} \sum_{i=0}^{n}\left(a_{i} \cdot \mathbf{1}_{S}(i)\right)+\lim _{n} \sum_{i=0}^{n}\left(a_{i} \cdot \mathbf{1}_{\omega \backslash S}(i)\right) \\
& =\sum_{i=0}^{\infty}\left(a_{i} \cdot \mathbf{1}_{S}(i)\right)+\sum_{i=0}^{\infty}\left(a_{i} \cdot \mathbf{1}_{\omega \backslash S}(i)\right) .
\end{aligned}
$$

We know that the equality marked with $(*)$ holds because the addition function, $+: X \times X \rightarrow X$, is continuous with respect to the norm topology on $X$ and the product topology on $X \times X$, and taking limits of convergent sequences commutes with applying continuous functions.

Lemma 3.7. Let $X$ be a Banach space, $\left(a_{i}\right)_{i \in \omega}$ a sequence in $X$, and $S \subseteq \omega$. Then the series $\Sigma\left(a_{i}\right)_{i \in \omega}$ absolutely converges if and only if the series $\Sigma\left(a_{i} \cdot \mathbf{1}_{S}(i)\right)_{i \in \omega}$ and $\Sigma\left(a_{i} \cdot \mathbf{1}_{\omega \backslash S}(i)\right)_{i \in \omega}$ absolutely converge. Moreover,

$$
\sum_{i=0}^{\infty} a_{i}=\sum_{i=0}^{\infty}\left(a_{i} \cdot \mathbf{1}_{S}(i)\right)+\sum_{i=0}^{\infty}\left(a_{i} \cdot \mathbf{1}_{\omega \backslash S}(i)\right) .
$$

Proof. Assume that the series $\Sigma\left(a_{i}\right)_{i \in \omega}$ absolutely converges. For all $i \in \omega$, we have $\left\|a_{i}\right\| \cdot \mathbf{1}_{S}(i) \leqslant\left\|a_{i}\right\|$ and $\left\|a_{i}\right\| \cdot \mathbf{1}_{\omega \backslash S}(i) \leqslant\left\|a_{i}\right\|$, so we conclude that the series $\Sigma\left(a_{i} \cdot \mathbf{1}_{S}(i)\right)_{i \in \omega}$ and $\Sigma\left(a_{i} \cdot \mathbf{1}_{\omega \backslash S}(i)\right)_{i \in \omega}$ absolutely converge. The converse follows by applying Lemma 3.6 to the sequence $\left(\left\|a_{i}\right\|\right)_{i \in \omega}$.

If all three series above absolutely converge, they also converge because $X$ is complete, so the desired equality follows directly from Lemma 3.6.

We additionally prove that the direct comparison test for series can be extended to well-ordered series.

Lemma 3.8. Let $X$ be a Banach space, $\alpha \in \omega_{1} \backslash \omega$, and $\left(a_{i}\right)_{i \in \alpha},\left(b_{i}\right)_{i \in \alpha}$ hypersequences in $X$ such that for all $i \in \alpha,\left\|a_{i}\right\| \leqslant\left\|b_{i}\right\|$. If the series $\Sigma\left(b_{i}\right)_{i \in \alpha}$ absolutely converges, then the series $\Sigma\left(a_{i}\right)_{i \in \alpha}$ absolutely converges and $\sum_{i \in \alpha}\left\|a_{i}\right\| \leqslant \sum_{i \in \alpha}\left\|b_{i}\right\|$. 
Proof. We prove the lemma by transfinite induction. If $\alpha=\beta+1$ for some ordinal $\beta$, from the induction hypothesis we have

$$
\sum_{i \in \alpha}\left\|a_{i}\right\|=\sum_{i \in \beta}\left\|a_{i}\right\|+\left\|a_{\beta}\right\| \leqslant \sum_{i \in \beta}\left\|b_{i}\right\|+\left\|b_{\beta}\right\|=\sum_{i \in \alpha}\left\|b_{i}\right\| .
$$

If $\alpha \in \operatorname{Lim}$, then for all $\beta \in \alpha$, we let $s_{\beta}:=\sum_{i \in \beta}\left\|a_{i}\right\|$ and compute

$$
s_{\beta} \leqslant \sum_{i \in \beta}\left\|b_{i}\right\| \leqslant \lim _{\beta \rightarrow \alpha} \sum_{i \in \beta}\left\|b_{i}\right\|=\sum_{i \in \alpha}\left\|b_{i}\right\| .
$$

Hence, the set of real numbers $\left\{s_{\beta}: \beta \in \alpha\right\}$ is bounded from above. Therefore, it has a supremum, which we denote with $L$. But then for all $\varepsilon>0$, there exists an $n_{\varepsilon} \in \omega$ such that $L-\varepsilon<a_{n_{\varepsilon}}$. For all $n \geqslant n_{\varepsilon}$, we then have $L-\varepsilon<s_{n_{\varepsilon}} \leqslant s_{n} \leqslant L<L+\varepsilon$. This implies $\left|s_{n}-L\right|<\varepsilon$, so the hypersequence $\left(s_{\beta}\right)_{\beta \in \alpha}$ converges.

We also know that $\lim _{\beta \rightarrow \alpha} s_{\beta} \leqslant \sum_{i \in \alpha}\left\|b_{i}\right\|$; otherwise, there would exist $i_{0} \in \alpha$ such that $a_{i_{0}}>b_{i_{0}}$.

Now that all of the preliminaries are in place, we can analyze the effects of reorderings on the convergence and sum of well-ordered series in Banach spaces. We devote the entirety of the following section to this topic, where we focus on absolutely convergent well-ordered series.

\section{EFFECTS of REORDERING ON ABSOLUte CONVERGENCE AND SUM}

In this section, we determine the effects of reorderings on the convergence and sum of absolutely convergent well-ordered series in Banach spaces.

Previously, we proved two simple lemmas that hinted at our general proof strategy; they let us separate a series into two parts, which can be summed individually, and the results added without changing the sum. In a proof by induction, one of the parts may be covered by the induction hypothesis, and what is left might be a single number, or an insignificant quantity. The former will be the case when we look at well-ordered series up to a successor ordinal; we will encounter the latter when we consider limit ordinals, where we make use of the fact that a convergent series has arbitrarily small tail sums. We also use the fact that for an absolutely convergent series $\Sigma a_{i}$, tail sums of the series $\Sigma\left\|a_{i}\right\|$ cannot be increased by leaving out some terms of $\Sigma\left\|a_{i}\right\|$.

By employing the strategy described in the previous paragraph, we first prove that an absolutely convergent standard series in a Banach space can be rearranged into a well-ordered series with respect to any countably infinite ordinal without affecting the convergence or sum of the series.

THEOREM 4.1. Let $\Sigma\left(a_{i}\right)_{i \in \omega}$ be an absolutely convergent series in a $B a$ nach space $X$. Then for all $\alpha \in \omega_{1} \backslash \omega$ and all bijections $f: \alpha \rightarrow \omega$, the 
well-ordered series $\Sigma\left(a_{f(i)}\right)_{i \in \alpha}$ absolutely converges and

$$
\sum_{i \in \alpha} a_{f(i)}=\sum_{i=0}^{\infty} a_{i}
$$

Proof (By TRANSFinite INDUCTION ON $\alpha$ ). If $\alpha=\omega$, the result is a basic theorem of mathematical analysis. Let $\alpha>\omega$ and let $f: \alpha \rightarrow \omega$ be a bijection. Suppose the statement is true for all ordinals $\beta \in \alpha \backslash \omega$. We separately consider two cases, where $\alpha$ is respectively a successor ordinal or a limit ordinal.

$\alpha=\beta+1$ We define the (hyper)sequence $\left(\hat{a}_{i}\right)_{i \in \omega}$ with

$$
\hat{a}_{i}:= \begin{cases}a_{i}, & \text { if } i<f(\beta) \\ a_{i+1}, & \text { if } i \geqslant f(\beta) .\end{cases}
$$

The function $g: \beta \rightarrow \omega$ defined with

$$
g(\gamma):= \begin{cases}f(\gamma), & \text { if } f(\gamma)<f(\beta) \\ f(\gamma)-1, & \text { if } f(\gamma)>f(\beta)\end{cases}
$$

is a bijection with the property $a_{f(i)}=\hat{a}_{g(i)}$ for all $i \in \beta$. From Lemma 3.7, we can now conclude

$$
\begin{aligned}
\sum_{i=0}^{\infty} a_{i} & =\sum_{i=0}^{\infty}\left(a_{i} \cdot \mathbf{1}_{\omega \backslash\{f(\beta)\}}(i)\right)+\sum_{i=0}^{\infty}\left(a_{i} \cdot \mathbf{1}_{\{f(\beta)\}}(i)\right)= \\
& =\sum_{i=0}^{\infty} \hat{a}_{i}+a_{f(\beta)}=\sum_{i \in \beta} \hat{a}_{g(i)}+a_{f(\beta)}=\sum_{i \in \beta} a_{f(i)}+a_{f(\beta)}=\sum_{i \in \alpha} a_{f(i)} .
\end{aligned}
$$

$\alpha \in \operatorname{Lim}$ For $\beta \in \alpha \backslash \omega$, we define sequences $\left(a_{i}^{\beta}\right)_{i \in \omega}$ and $\left(\bar{a}_{i}^{\beta}\right)_{i \in \omega}$ with

$$
\begin{aligned}
a_{i}^{\beta} & :=a_{i} \cdot \mathbf{1}_{f[\beta]}(i) \text { and } \\
\bar{a}_{i}^{\beta} & :=a_{i} \cdot \mathbf{1}_{f[\alpha \backslash \beta]}(i),
\end{aligned}
$$

where $f[\beta]$ denotes the set $\{f(\gamma): \gamma \in \beta\}$. As in the previous case, we use Lemma 3.7 to conclude

$$
\sum_{i=0}^{\infty} a_{i}=\sum_{i=0}^{\infty} a_{i}^{\beta}+\sum_{i=0}^{\infty} \bar{a}_{i}^{\beta} .
$$

For an infinite well-ordered set $(S, \leqslant)$ and all $n \in \omega$, we denote the set obtained by removing the first $n$ elements from $S$ with $S_{n}$. More precisely, we define

$$
S_{0}=S ; \quad S_{n+1}=S_{n} \backslash\left\{\min \left(S_{n}\right)\right\} .
$$


$\begin{array}{ccccccccccc}f[\beta] & a_{0} & a_{1} & a_{2} & \ldots & a_{n_{0}} & & a_{n_{0}+2} & a_{n_{0}+3} & & \cdots \\ f[\alpha \backslash \beta] & & & & & & a_{n_{0}+1} & & & a_{n_{0}+4} & \ldots\end{array}$

FiguRE 1. When $\alpha$ is a limit ordinal, we split the series $\Sigma a_{i}$ in two parts: one with terms indexed by $f[\beta]$ that contains the "significant" part of the sum, and the "small" rest indexed by $f[\alpha \backslash \beta]$.

We use this notation to define a bijection $g: \beta \rightarrow \omega$ that assigns to each ordinal $i \in \beta$ the position of its image $f(i)$ in the well-ordered set $f[\beta]$ with

$$
g:=\left\{(i, n) \in \beta \times \omega: f(i)=\min \left(f[\beta]_{n}\right)\right\} .
$$

In other words, for infinite $f[\beta]=\left\{f\left(i_{0}\right)<f\left(i_{1}\right)<f\left(i_{2}\right)<\cdots\right\} \subseteq \omega$, we set $g\left(i_{0}\right):=0, g\left(i_{1}\right):=1, g\left(i_{2}\right):=2$, etc. Note that for all $i \in \beta, a_{f(i)}=a_{g(i)}^{\beta}$ and

$$
g(i)=\operatorname{card}\{k \in f[\beta]: k<f(i)\} .
$$

From the induction hypothesis and $(*)$ we get

$$
\sum_{i \in \beta} a_{f(i)}=\sum_{i \in \beta} a_{g(i)}^{\beta}=\sum_{i=0}^{\infty} a_{i}^{\beta}=\sum_{i=0}^{\infty} a_{i}-\sum_{i=0}^{\infty} \bar{a}_{i}^{\beta} .
$$

The idea now is to split the series as shown in Fig. 1. To that end, let $\varepsilon>0$. We are looking for an ordinal $\beta_{0} \in \alpha$ such that for all $\beta \in \alpha \backslash \beta_{0}$, $\left\|\sum_{i \in \beta} a_{f(i)}-\sum_{i=0}^{\infty} a_{i}\right\|<\varepsilon$. Let $n_{0} \in \omega$ such that $\sum_{i=n_{0}}^{\infty}\left\|a_{i}\right\|<\varepsilon$. We define

$$
\begin{aligned}
\beta_{0} & :=\min \left(\left\{\gamma \in \alpha: \gamma>\omega \wedge\left(\forall \gamma^{\prime} \in \alpha\right)\left(\gamma^{\prime} \geqslant \gamma \rightarrow f\left(\gamma^{\prime}\right)>n_{0}\right)\right\}\right) \\
& =\max \left(\{\omega\} \cup f^{-1}\left[n_{0}+1\right]\right)+1<\alpha .
\end{aligned}
$$

For all $\beta \in \alpha \backslash \beta_{0}$, due to Lemma 3.8 we have

$$
\left\|\sum_{i \in \beta} a_{f(i)}-\sum_{i=0}^{\infty} a_{i}\right\|=\left\|\sum_{i=0}^{\infty} \bar{a}_{i}^{\beta}\right\| \leqslant \sum_{i=0}^{\infty}\left\|\bar{a}_{i}^{\beta}\right\| \leqslant \sum_{i=n_{0}}^{\infty}\left\|a_{i}\right\|<\varepsilon .
$$

Now we consider what happens when going the other way; we prove that we can safely reorder an absolutely convergent well-ordered series in a Banach space into a standard series. We will actually be able to leverage Theorem 4.1 in the proof by employing a suitable choice of reordering.

THEOREM 4.2. Let $\alpha \in \omega_{1} \backslash \omega$ and let $\Sigma\left(a_{i}\right)_{i \in \alpha}$ be an absolutely convergent well-ordered series in a Banach space $X$. Then for all bijections $f: \omega \rightarrow \alpha$, the series $\Sigma\left(a_{f(i)}\right)_{i \in \omega}$ absolutely converges and

$$
\sum_{i=0}^{\infty} a_{f(i)}=\sum_{i \in \alpha} a_{i}
$$


Proof. For every $n \in \omega$, we denote the $n$-th partial sum of the series $\Sigma\left(\left\|a_{f(i)}\right\|\right)_{i \in \omega}$ with $s_{n}$ and compute

$$
s_{n}=\sum_{i \in \omega}\left\|a_{f(i)}\right\| \cdot \mathbf{1}_{n}(i)=\sum_{i \in \alpha}\left\|a_{i}\right\| \cdot \mathbf{1}_{n}\left(f^{-1}(i)\right) \leqslant \sum_{i \in \alpha}\left\|a_{i}\right\|,
$$

where the last inequality can be obtained directly from Lemma 3.8. Therefore, $\left(s_{n}\right)_{n \in \omega}$ is a monotonically increasing sequence of real numbers that is bounded from above, so it converges.

To prove the second part of the theorem, let $\hat{a}_{i}:=a_{f(i)}$ for all $i \in \omega$. We know $f^{-1}: \alpha \rightarrow \omega$ is a bijection, so Theorem 4.1 gives us

$$
\sum_{i=0}^{\infty} a_{f(i)}=\sum_{i=0}^{\infty} \hat{a}_{i}=\sum_{i \in \alpha} \hat{a}_{f^{-1}(i)}=\sum_{i \in \alpha} a_{f\left(f^{-1}(i)\right)}=\sum_{i \in \alpha} a_{i} .
$$

The two theorems can now be combined to prove that any reordering, i.e., a bijection between countably infinite ordinals, of an absolutely convergent well-ordered series in a Banach space yields another absolutely convergent well-ordered series where the initial sum is preserved.

Corollary 4.3. Let $\alpha, \beta \in \omega_{1} \backslash \omega$ and let $\Sigma\left(a_{i}\right)_{i \in \alpha}$ be an absolutely convergent well-ordered series. Then for all bijections $f: \beta \rightarrow \alpha$, the wellordered series $\Sigma\left(a_{f(i)}\right)_{i \in \beta}$ absolutely converges and

$$
\sum_{i \in \beta} a_{f(i)}=\sum_{i \in \alpha} a_{i}
$$

Proof. Let $g: \omega \rightarrow \alpha$ be a bijection. Then $\left(g^{-1} \circ f\right): \beta \rightarrow \omega$ is also a bijection. If we define $\hat{a}_{i}:=a_{g(i)}$ for all $i \in \omega$, from Theorems 4.1 and 4.2 we get

$$
\sum_{i \in \alpha} a_{i}=\sum_{i=0}^{\infty} a_{g(i)}=\sum_{i=0}^{\infty} \hat{a}_{i}=\sum_{i \in \beta} \hat{a}_{\left(g^{-1} \circ f\right)(i)}=\sum_{i \in \beta} a_{f(i)} .
$$

\section{Conclusion}

In this paper, we wanted to take a step towards a general treatise of the effects of reorderings on the convergence and sum of well-ordered series. Our main focus was on reordering absolutely convergent well-ordered series in Banach spaces. Such reorderings often occur in mathematical practice, so identifying a general underlying principle is of practical as well as theoretical interest.

To define the notion of well-ordered series itself, we followed the modern way of employing transfinite recursion over the structure of the index set. Our chosen setting of Banach spaces may be possible to improve upon when absolute convergence is replaced by a different kind of convergence; in the future, we would like to extend our analysis to more general uniform spaces, such as Hausdorff topological abelian groups, and investigate in detail the 
effects of reorderings on the conditional and unconditional convergence of well-ordered series in such a setting.

\section{ACKNOWLEDGEMENTS.}

We would like to thank Zvonimir Šikić for introducing the problem of rearranging well-ordered series to us. We also thank Siniša Miličić, Filip Nikšić, Zvonimir Sviben, Domagoj Vrgoč and Mladen Vuković for helpful comments and discussions.

\section{REFERENCES}

[1] J. P. Aguilera and D. Fernández-Duque, Strong completeness of provability logic for ordinal spaces, J. Symb. Log. 82 (2017), 608-628.

[2] J. Dieudonné, Foundations of Modern Analysis. Academic Press, 1960.

[3] A. Dvoretzky and C. A. Rogers, Absolute and unconditional convergence in normed linear spaces, Proc. Nat. Acad. Sci. U. S. A. 36 (1950), 192-197.

[4] R. L. Graham, D. E. Knuth and O. Patashnik, Concrete Mathematics: A Foundation for Computer Science. Addison-Wesley Longman Publishing Co., Inc., Boston, MA, USA, 2nd edition, 1994.

[5] S. V. Heikkilä, On summability, integrability and impulsive differential equations in Banach spaces, Bound. Value Probl. 2013, 2013:91.

[6] T. H. Hildebrandt, On unconditional convergence in normed vector spaces, Bull. Amer. Math. Soc. 46 (1940), 959-962.

[7] P. Komjath, Rearranging transfinite series of ordinals, Bull. Austral. Math. Soc. 16 (1977), 321-323.

[8] J. C. Shepherdson, Well-ordered sub-series of general series, Proc. London Math. Soc. (3) 1 (1951), 291-307.

\section{Promjena redoslijeda članova kod apsolutno konvergentnih dobro uređenih redova u Banachovim prostorima}

\section{Vedran Čačić, Marko Doko i Marko Horvat}

SAŽETAK. Nalazimo općenit princip promjene redoslijeda članova za dobro uređene redove u Banachovim prostorima. Dokazujemo da za svaki apsolutno konvergentan dobro uređen red indeksiran prebrojivim ordinalom, red s istim članovima indeksiran bilo kojim prebrojivim ordinalom također apsolutno konvergira i ima istu sumu. 
Vedran Čačić

Department of Mathematics

University of Zagreb

10000 Zagreb, Croatia

E-mail: veky@math.hr

Marko Doko

Max Planck Institute for Software Systems

67663 Kaiserslautern, Germany

E-mail: mdoko@mpi-sws.org

Marko Horvat

Department of Mathematics

University of Zagreb

10000 Zagreb, Croatia

E-mail: mhorvat@math.hr

Received: 23.2.2019.

Accepted: 16.4.2019. 
